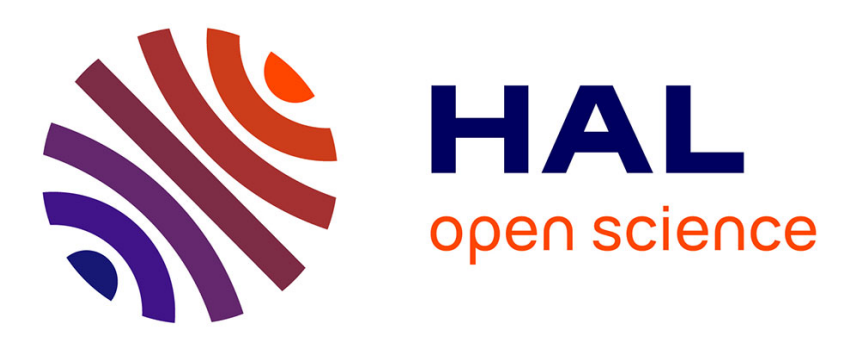

\title{
Robust time-sub-optimal control of the saturated double integrator applied to attitude stabilization
}

\author{
Mirko Brentari, Paolo Bosetti, Rafal Goebel, Luca Zaccarian
}

\section{To cite this version:}

Mirko Brentari, Paolo Bosetti, Rafal Goebel, Luca Zaccarian. Robust time-sub-optimal control of the saturated double integrator applied to attitude stabilization. 2017 IEEE 56th Annual Conference on Decision and Control (CDC), Dec 2017, Melbourne, Australia. 10p., 10.1109/CDC.2017.8264472 . hal-01700161

\section{HAL Id: hal-01700161 \\ https://hal.laas.fr/hal-01700161}

Submitted on 19 Feb 2018

HAL is a multi-disciplinary open access archive for the deposit and dissemination of scientific research documents, whether they are published or not. The documents may come from teaching and research institutions in France or abroad, or from public or private research centers.
L'archive ouverte pluridisciplinaire HAL, est destinée au dépôt et à la diffusion de documents scientifiques de niveau recherche, publiés ou non, émanant des établissements d'enseignement et de recherche français ou étrangers, des laboratoires publics ou privés. 


\title{
Robust time-sub-optimal control of the saturated double integrator applied to attitude stabilization
}

\author{
Mirko Brentari, Paolo Bosetti, Rafal Goebel, and Luca Zaccarian
}

\begin{abstract}
This paper proposes a robust time-sub-optimal controller for the double integrator with saturated input, based on a hybrid blend of a local and a global controller. The scheme makes use of two hysteresis mechanisms in order to provide robustness to unmodelled dynamics and measurement noise. Rigorous certificates of the stability of the proposed controller are given, by exploiting the properties of hybrid dynamical system established in recent works of Teel et al. Attitude stabilization with the proposed control strategy is then illustrated by simulations. The method shows its effectiveness in stabilizing the attitude, preventing the typical chattering and robustness issues emerging with classical time-optimal implementations.
\end{abstract}

\section{INTRODUCTION}

Chains of integrators with saturated input are fundamental dynamical processes. The control of them is widely investigated by the control community, see [1], [2], etc. Among many control strategies, timeoptimal controllers are capable of a finite time stabilization of such plants in minimum time, see for example [3], [4].

Time-optimal control with bounded controls is well-known to yield bang-bang and hence discontinuous open-loop and feedback controls [5]. A classical example of this is the second-order integrator $\dot{x}_{1}=u, \dot{x}_{2}=x_{1}$, with $u \in[-1,1]$, which is one of the most fundamental systems in control applications, and has many mechatronic applications [6] [7]. There, the time-optimal feedback that drives states to the origin takes on the value -1 above the curve $x_{2}=\operatorname{sign} x_{1} x_{1}^{2} / 2$, and the value 1 below that curve, and is thus discontinuous along that curve. Open-loop controls switch from one value of the control to its negative upon reaching this "switching curve". The computation of the curve, thanks to the Pontryagin Maximum Principle, boils down to backward integration of the optimal dynamics, and the same idea applies to higher-order integrators. More advanced methods, for example Groebner bases, have been tested in computation of the switching surfaces for the third-order integrator [8] and can be used for more involved linear dynamics [9].

The discontinuity of the time-optimal feedback raises the issue of its robustness. Essentially, the discontinuity, measurement error and/or unmodelled dynamics may lead to frequent switching/chattering and loss of performance. One early proposal to deal with this [10], for the second-order integrator, involves smoothing of the bang-bang behavior along the switching curve and linear control near the origin, resulting in sub-optimal behavior. Similarly, replacing the switching curve and the two regions, above and below it, by three regions has been considered in discrete-time [7] and applied to diskdrive control. Similar ideas are followed for the third-order integrator in [2], [11]. Alternatively, some robustness can be introduced through hysteresis [12], suggesting a hybrid formulation of the feedback. For a related discussion of robustness etc. aspects of time-optimal and other stabilizing controllers for the second-order integrator, see [13].

This paper builds on the ideas considered before, like hysteresis implementation of the switching surface and application of linear feedback near the origin, and, for the second-order integrator, formulates

M. Brentari, P. Bosetti and L. Zaccarian are with Dipartimento di Ingegneria Industriale, University of Trento, Italy.

L. Zaccarian is with LAAS-CNRS, Université de Toulouse, CNRS, France. Research funded in part by grant PowerLyap funded by CaRiTRo.

R. Goebel is with Department of Mathematics and Statistics, Loyola University Chicago, 1032 W. Sheridan Road, Chicago, IL 60660. Email: rgoebel1@luc.edu. This work was partially supported by the Simons Foundation Grant 315326. 
a sub-time-optimal feedback in the hybrid dynamical system framework of [14]. The use of hybrid controllers for improving performances of continuos time plants have been already investigated for example in [15] and, for reset control systems, in [16]. One advantage of using this framework is that it easily allows for modeling of hysteresis-type switching of various controllers, for example a local (in this case, linear) and a global one; see [14] and, for example, [17]. The resulting model is, in fact, simpler than one resulting from continuous interpolation of the original discontinuous feedback. Another advantage is that robustness of asymptotic stability follows from general results in [14], if the data meets mild regularity conditions (the functions modeling the feedback are continuous, and the set where different functions apply are closed).

The paper is organized as follows. Section II discusses the switching sets needed for the synthesis of the time-optimal control of the saturated double integrator. Section III uses these sets to design a hybrid sub-time-optimal feedback control law with hysteresis. Section IV describes the blending of this sub-time-optimal control with a local linear feedback. In Section V, an application of the designed control law to a 3D attitude dynamics is presented. Section VI draws some conclusions and presents future perspectives of this work. All the proofs are omitted due to space constraints.

\section{SWITCHING SETS}

Let us consider the following double integrator dynamical system with state $x=\left[x_{1} x_{2}\right]^{\top} \in \mathbb{R}^{2}$ :

$$
\dot{x}=\left[\begin{array}{c}
\dot{x}_{1} \\
\dot{x}_{2}
\end{array}\right]=\left[\begin{array}{c}
q \\
x_{1}
\end{array}\right]=: A x+b q=\underbrace{\left[\begin{array}{ll}
0 & 0 \\
1 & 0
\end{array}\right]}_{:=A} x+\underbrace{\left[\begin{array}{c}
1 \\
0
\end{array}\right]}_{:=b} q
$$

together with bounded input $q$ :

$$
q \in[-1,1]
$$

\section{A. Implicit Formulation}

Let $\tau_{m}$ be a sequence of times:

$$
\tau_{m}:=\left(t_{1}, t_{2}, \ldots, t_{m}\right)
$$

and $\mathcal{T}_{m}$ be the set of any $\tau_{m}$ such that $\tau_{m}$ is a sequence of nondecreasing times:

$$
\mathcal{T}_{m}:=\left\{\tau_{m} \mid 0 \leq t_{1} \leq t_{2} \leq \cdots \leq t_{m}\right\}
$$

Definition 1: From [18], given $\tau_{m} \in \mathcal{T}_{m}$, let $\mathcal{O}_{m+}$ (respectively, $\mathcal{O}_{m-}$ ) be the set of states $x$ for which there exists $\tau_{m}=\left(t_{1}, t_{2}, \ldots, t_{m}\right) \in \mathcal{T}_{m}$, such that the backwards solution $x_{b}$ to (1) starting at the origin, with input $q$ toggling between -1 and 1 at (backwards) times $-t_{i}, i=1, \ldots, m$, and finally toggling from $x_{b}\left(-t_{m}^{-}\right)=1$ to $x_{b}\left(-t_{m}^{+}\right)=-1$, reaches the point $x_{b}\left(-t_{m}\right)=x_{b}\left(-t_{m}^{-}\right)=x_{b}\left(-t_{m}^{+}\right)=x$.

By definition, we assume:

$$
\mathcal{O}_{0+}:=\mathcal{O}_{0-}:=\{0\}
$$

Sets $\mathcal{O}_{m+}$ and $\mathcal{O}_{m+}$ can be computed applying recursively the variation of constants formula [19, Chap. 1.6] to dynamics (1) in reverse time starting from $\mathcal{O}_{0}$ (i.e., the origin).

We define $\mathcal{O}_{m}$ as:

$$
\mathcal{O}_{m}:=\mathcal{O}_{m-} \cup \mathcal{O}_{m+}
$$

Theorem 1: ([18, Theorem 5.4]): The system (1) has, for every initial condition, a unique time-optimal control, which is bang-bang (i.e., it takes only the extreme values) and switches at most 1 time.

Theorem 1 implies, among other things, the following:

Proposition 1: Let us define the following sets:

$$
\mathcal{U}_{+}:=\mathcal{O}_{2+} \backslash \mathcal{O}_{1}, \mathcal{U}_{-}:=\mathcal{O}_{2-} \backslash \mathcal{O}_{1}
$$


Then, given a generic initial condition $x_{0}$ for system (1), $x_{0} \in \mathcal{U}_{+}$(respectively, $x_{0} \in \mathcal{U}_{-}$) implies that the time optimal control takes the value $q=-1$ (respectively, $q=1$ ) for an initial time interval of non zero length.

Moreover, it holds that $\mathcal{U}_{+} \cap \mathcal{U}_{-}=\varnothing$ and

$$
\overline{\mathcal{U}_{+} \cup \mathcal{U}_{-}}=\mathbb{R}^{2}
$$

Proposition 1 essentially establishes that sets $\mathcal{U}_{+}$and $\mathcal{U}_{-}$are a pair of regions covering almost all the state-space of plant (1) where it is straightforward to make the right selection of input $q$ towards a time-optimal stabilization of the origin. In particular, it is underlined that the complement of $\mathcal{U}_{+} \cup \mathcal{U}_{-}$ (namely $\mathcal{O}_{1}$ ) corresponds to a set of measure zero wherein the time-optimal selection of input $q$ becomes more involved and requires distinguishing between multiple nontrivial subcases.

This paper provides an illustration for the double integrator of a suggestive feedback control approach where we only specify the feedback law in $\mathcal{U}_{+} \cup \mathcal{U}_{-}$(or a slightly smaller set), where the feedback law easily comes from Proposition 1 , and to define a hysteresis zone in a slightly inflated region around $\mathcal{O}_{1}$ wherein, rather than understanding the exact time-optimal feedback, we make use of a memory variable persisting with the feedback action previously selected. The advantage of this approach is that it does not require to determine the value of the time optimal input in $\mathcal{O}_{1}$.

\section{B. Representation of $\mathcal{U}_{+}$and $\mathcal{U}_{-}$}

In this section, we will provide an explicit formulation of $\mathcal{U}_{+}$and $\mathcal{U}_{-}$. By backward integration of the dynamics (1), it is possible to develop

$$
\begin{aligned}
& \mathcal{O}_{1+}=\bigcup_{t_{1} \geq 0}\left\{\left[-t_{1}, \frac{t_{1}^{2}}{2}\right]^{\top}\right\}=\bigcup_{x_{1} \leq 0}\left\{\left[x_{1}, \frac{x_{1}^{2}}{2}\right]^{\top}\right\} \\
& \mathcal{O}_{1-}=\bigcup_{t_{1} \geq 0}\left\{\left[t_{1},-\frac{t_{1}^{2}}{2}\right]^{\top}\right\}=\bigcup_{x_{1} \geq 0}\left\{\left[x_{1},-\frac{x_{1}^{2}}{2}\right]^{\top}\right\} .
\end{aligned}
$$

An explicit representation is then

$$
\begin{aligned}
& \mathcal{O}_{1+}=\{x \in \mathbb{R}^{2} \mid \underbrace{x_{2}-\frac{x_{1}^{2}}{2}}_{:=\varphi_{+}(x)}=0, x_{1} \leq 0\} \\
& \mathcal{O}_{1-}=\{x \in \mathbb{R}^{2} \mid \underbrace{x_{2}+\frac{x_{1}^{2}}{2}}_{:=\varphi_{-}(x)}=0, x_{1} \geq 0\} .
\end{aligned}
$$

It is now straightforward to express the union $\mathcal{O}_{1}$ as

$$
\mathcal{O}_{1}:=\mathcal{O}_{1+} \cup \mathcal{O}_{1-}=\left\{x \in \mathbb{R}^{2} \mid \varphi(x)=0\right\},
$$

where

$$
\varphi(x):= \begin{cases}\varphi_{+}(x), & \text { if } x_{1} \leq 0 \\ \varphi_{-}(x), & \text { if } x_{1} \geq 0 .\end{cases}
$$

is continuous since $\varphi_{+}\left(\left[\begin{array}{c}0 \\ x_{2}\end{array}\right]\right)=\varphi_{-}\left(\left[\begin{array}{c}0 \\ x_{2}\end{array}\right]\right), \forall x_{2}$. Following the same procedure (backward integration), $\mathcal{O}_{2+}$ and $\mathcal{O}_{2-}$ can now be computed based on

$$
\mathcal{T}_{2}=\left\{\tau_{2} \mid 0 \leq t_{1} \leq t_{2}\right\}
$$


as follows:

$$
\begin{aligned}
& \mathcal{O}_{2+}=\bigcup_{t_{2} \geq t_{1} \geq 0}\left\{\left[t_{2}-2 t_{1},-\frac{t_{2}^{2}}{2}-t_{1}^{2}+2 t_{1} t_{2}\right]^{\top}\right\} \\
& \mathcal{O}_{2-}=\bigcup_{t_{2} \geq t_{1} \geq 0}\left\{\left[2 t_{1}-t_{2}, \frac{t_{2}^{2}}{2}+t_{1}^{2}-2 t_{1} t_{2}\right]^{\top}\right\} .
\end{aligned}
$$

Some computations show that

$$
\mathcal{O}_{2+}=\left\{x \in \mathbb{R}^{2} \mid \varphi(x) \geq 0\right\}, \mathcal{O}_{2-}=\left\{x \in \mathbb{R}^{2} \mid \varphi(x) \leq 0\right\} .
$$

Based on these considerations, for $n=2$ it holds that

$$
\mathcal{U}_{+}=\left\{x \in \mathbb{R}^{2} \mid \varphi(x)>0\right\}, \mathcal{U}_{-}=\left\{x \in \mathbb{R}^{2} \mid \varphi(x)<0\right\} .
$$

\section{Hybrid FramewORK FOR Robust Time-Optimal CONTROL}

In this section we exploit Proposition 1 and description (13) to design an $\epsilon$-modification of the feedback law ensuring global convergence to an $\epsilon$-small neighborhood of the origin for the double integrator (1).

Rather then treating $q$ in (1) as an input, we perform this by choosing an overall state:

$$
\xi:=\operatorname{col}(x, q) \in \mathbb{R}^{2} \times\{-1,1\}
$$

and note that $x \in \mathcal{U}_{+}$and $q=1$ (respectively, $x \in \mathcal{U}_{-}$and $q=-1$ ) implies that $q$ should toggle. Consequently, we represent our hybrid stabilizer in terms of the set $\mathcal{D}_{0}$ from which $q$ should toggle between +1 and -1 via the trivial jump map $q^{+}=-q$. In particular, based on Propositions 1 and description (13), one notices that both in $\mathcal{U}_{+}$and in $\mathcal{U}_{-}$, the product $q \varphi(x)$ is always negative, and therefore that such a set $\mathcal{D}_{0}$ could be selected as:

$$
\mathcal{D}_{0}=\{\xi \mid \varphi(x) q>0\},
$$

which is not a closed set. Since we are interested in robust stabilizers, and [14] shows that closed jump/flow sets ensure robustness of stability, rather than selecting $\mathcal{D}_{0}$ in (15), we prefer to introduce a hysteresis mechanism related to the choice of a (small) scalar $\epsilon>0$ and the following $\epsilon$-dependent closed loop:

$$
\begin{aligned}
& \dot{\xi}=A_{c} \xi \quad \xi \in \mathcal{C}:=\{\xi \mid \varphi(x) q \leq \epsilon\} \\
& \xi^{+}=A_{d} \xi \quad \xi \in \mathcal{D}:=\{\xi \mid \varphi(x) q \geq \epsilon\}
\end{aligned}
$$

where, with $I_{2}$ being the $2 \times 2$ identity matrix,

$$
\begin{aligned}
& A_{c}:=\left[\begin{array}{c|c}
A & b \\
\hline 0 & 0
\end{array}\right] \in \mathbb{R}^{3 \times 3} \\
& A_{d}:=\left[\begin{array}{c|c}
I_{2} & 0 \\
\hline 0 & -1
\end{array}\right] \in \mathbb{R}^{3 \times 3} .
\end{aligned}
$$

In system (16), matrix $A_{c}$ simply encodes equation (1a), while $A_{d}$ encodes the toggling mechanism of $q$. Finally, as commented above, set $\mathcal{D}$ encodes the fact that a switch should happen whenever $q \varphi(x)>0$ (because this means that $x \in \mathcal{U}_{-} \cup \mathcal{U}_{+}$and $q$ has the wrong sign), possibly allowing for some "erroneous" feedback when $|\varphi(x)| \leq \epsilon$.

Remark 1: The advantage of this $\epsilon$-modified law is to introduce a hysteresis zone around $\mathcal{O}_{1}$, the set where characterizing the time-optimal feedback could require extra care. Indeed, the selection of a positive $\epsilon$ gives several advantages. It permits to synthesize a time-sub-optimal control law based on the knowledge of only the two sets $\mathcal{U}_{+}$and $\mathcal{U}_{-}$. Moreover, it prevents the possibility of Zeno solutions (i.e., 
solutions with persisting jumps) [14, Definition 2.5], corresponding to having a control $q$ that switches infinitely fast, causing a chattering phenomena and possibly damage of the actuators. Moreover, this $\epsilon$-inflation makes the controller robust to unmodeled dynamics or sufficiently small measurement noise. The smaller is the hysteresis amplitude $\epsilon$, the closer the input is to the optimal one, but the less robust is the control scheme. Therefore, the parameter $\epsilon$ represents a controller tuning parameter, acting as a trade-off between time optimality and robustness.

For clarity, the phase portrait of system (16) is depicted in Figure 1.
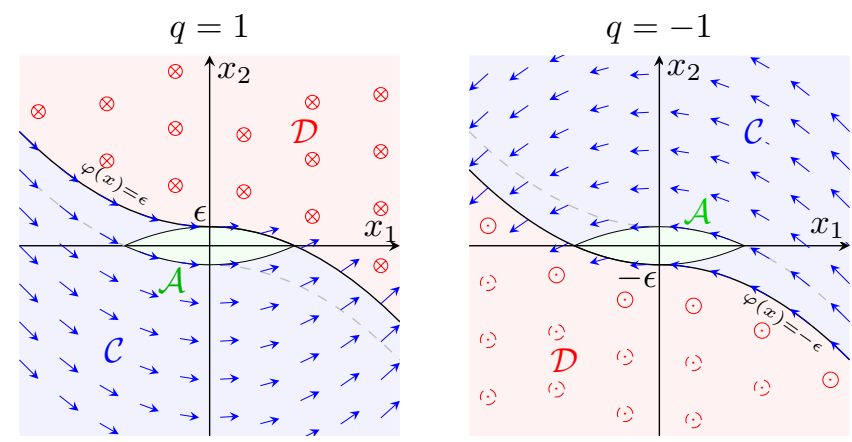

Fig. 1: Flow set $\mathcal{C}$ and jump set $\mathcal{D}$ and phase portrait of (16).

Observe that the hysteresis introduced by the parameter $\epsilon>0$ introduces a neighborhood of $\mathcal{O}_{1}$ where solutions can flow assuming any of the two values of $q$. All solutions exhibit an "eye-shaped" steady-state limit cycle whose orbit can be described by:

$$
\lambda:=\{x \mid v(x)=\epsilon\}
$$

where $v(x)$ is the following scalar function:

$$
v(x):=\left\{\begin{array}{ll}
\frac{x_{1}^{2}}{2}+x_{2} & \text { if } x_{2} \geq 0 \\
\frac{x_{1}^{2}}{2}-x_{2} & \text { if } x_{2} \leq 0
\end{array},\right.
$$

Stability properties is established for a set where the input $q$ is "don't care" and the state $x$ belongs to some compact neighborhood $\mathcal{A}_{x}$ of the origin. In particular, our main result below establishes stability properties of the following bounded attractor, also depicted in green in Figure 1:

$$
\mathcal{A}:=\mathcal{A}_{x} \times\{-1,1\}:=\{x \mid v(x) \leq \epsilon\} \times\{-1,1\} .
$$

Theorem 2: Attractor (19) is UGAS for (16).

\section{BLEND WITH A LOCAL LINEAR FEEDBACK}

In this section, the blending of the controller developed in Section III with a local linear feedback is addressed. The local controller is chosen as a linear a state feedback $k x$ inducing the closed loop:

$$
\dot{x}=A_{c l} x:=\left(A+b k^{\top}\right) x,
$$

where $k \in \mathbb{R}^{2}$ can be any gain vector such that matrix $A_{c l}$ is Hurwitz. For example, vector $k$ can be selected as an LQR gain.

This controller can be incorporated in hybrid system (16) by modifying the matrix $A_{c}$ previously defined in (16b) as follows:

$$
A_{c}=\left[\begin{array}{c|c}
A+(1-|q|) b k^{\top} & b \\
\hline 0 & 0
\end{array}\right] \in \mathbb{R}^{3 \times 3} .
$$


With the latter, the local controller is activated whenever the logic variable $q$ is set to zero. In particular, the new dynamics evolves in the set $\xi \in \mathbb{R}^{n} \times\{-1,0,1\}$ extending the previous set $\xi \in \mathbb{R}^{n} \times\{-1,1\}$ in (16).

A strategy for choosing whether the local controller or the global controller of Section III has to be applied is needed. For this purpose, the uniting global and local controller strategy presented in [14, Example 1.7] and [17] is adopted, where we use $q$ as a supervisor variable. In particular, when $|q|=1$ the global controller is active, whereas when $q=0$ the local controller is activated by virtue of (21). Following [14, Example 1.7], given scalars $0<\rho<1$ and $\ell \in \mathbb{R}_{+}$to be chosen later, if $P=P^{\top}>0$ is any matrix solving the continuous time Lyapunov equation

$$
\left(A+b k^{\top}\right)^{\top} P+P\left(A+b k^{\top}\right)<0,
$$

we define the set where we would like to switch from the local to the global controller as:

$$
\mathcal{D}_{l}:=\left\{\xi \mid q=0, x^{\top} P x \geq \ell\right\} .
$$

Also, we define the set where we would like to switch from the global to the local controller as:

$$
\mathcal{D}_{g}:=\left\{\xi|| q \mid=1, x^{\top} P x \leq \rho \ell\right\} .
$$

The overall jump dynamics is the following one:

$$
\xi^{+} \in G(\xi), \quad \xi \in \mathcal{D}_{u}:=\mathcal{D}_{l} \cup \mathcal{D}_{g} \cup \mathcal{D},
$$

where the jump map $G(\xi)$ corresponds to:

$$
\begin{aligned}
& G(\xi)= \\
& = \begin{cases}\left\{\left[\begin{array}{c}
x \\
\pm(1-|q|)
\end{array}\right]\right\} & \text { if } \xi \in\left(\mathcal{D}_{l} \cup \mathcal{D}_{g}\right) \backslash \mathcal{D} \\
{\left[\begin{array}{c}
x \\
-q
\end{array}\right]} & \text { if } \xi \in \mathcal{D} \backslash\left(\mathcal{D}_{l} \cup \mathcal{D}_{g}\right) \\
\left\{\left[\begin{array}{c}
x \\
\pm(1-|q|)
\end{array}\right]\right\} \cup\left\{\left[\begin{array}{c}
x \\
-q
\end{array}\right]\right\} & \text { if } \xi \in \mathcal{D} \cap\left(\mathcal{D}_{l} \cup \mathcal{D}_{g}\right),\end{cases}
\end{aligned}
$$

where $\left\{\left[\begin{array}{c}x \\ \pm(1-|q|)\end{array}\right]\right\}:=\left\{\left[\begin{array}{c}x \\ (1-|q|)\end{array}\right]\right\} \cup\left\{\left[\begin{array}{c}x \\ -(1-|q|)\end{array}\right]\right\}$. The overall flow equation is enabled on the closed complement of $\mathcal{D}_{u}$, and from (16) corresponds to:

$$
\dot{\xi}=A_{c} \xi, \quad \xi \in \mathcal{C}_{u}:=\overline{\mathbb{R}^{n} \times\{-1,0,1\} \backslash \mathcal{D}_{u}},
$$

with $A_{c}$ defined in equation (21).

The proposed scheme is completed by a suitable selection of the scalars $\ell$ and $\rho$ in (23) ensuring that the local feedback $k^{\top} x$ has smaller norm than 1 (i.e., it is below the saturation level) as long as $x^{\top} P x \leq \ell$. This property is established in the next lemma.

Lemma 1: Given $P=P^{\top}>0$ and a gain $k \in \mathbb{R}^{n}$, if $\ell=\left(k^{\top} P^{-1} k\right)^{-1}$, then

$$
x^{\top} P x \leq \ell \Rightarrow\left|k^{\top} x\right| \leq 1
$$

Based on Lemma 1, we can prove the following statement, which requires that set $\mathcal{A}$ is sufficiently small ( $\epsilon$ is sufficiently small) or scalar $\ell$ is sufficiently large ( $k$ is sufficiently small).

Theorem 3: Given $P$ and $k$ satisfying (22) and $\ell=\left(k^{\top} P^{-1} k\right)^{-1}$, if $\rho \in(0,1)$ and $\epsilon$ are such that $\mathcal{A} \subset \operatorname{int}\left(\mathcal{D}_{g}\right)$ and $\mathcal{A}$ is UGAS for system (16), then $A_{0}:=\{0\} \times\{-1,0,1\}$ is UGAS for system (24). 
To check the condition $\mathcal{A} \subset \operatorname{int}\left(\mathcal{D}_{g}\right)$ we may use an auxiliary bounding box $\mathcal{B}$ of the attractor $\mathcal{A}$ (such that $\mathcal{A} \subset \mathcal{B}$ ) such that $\mathcal{B} \subset \operatorname{int}\left(\mathcal{D}_{g}\right)$ is easier to check. In particular, according to Figure 2 , we suggest the following selection, whose effectiveness is established in the corollary below.

$$
\mathcal{B}:=\left\{\xi|| x_{1}|<\sqrt{2 \epsilon},| x_{2} \mid<\epsilon\right\}
$$

Corollary 1: Given $P$ and $k$ satisfying (22) and $\ell=\left(k^{\top} P^{-1} k\right)^{-1}$, if

$$
\max \left\{a^{\top} P a, b^{\top} P b\right\}<\rho \ell
$$

with $a=[\sqrt{2 \epsilon}, \epsilon]^{\top}$ and $b=[\sqrt{2 \epsilon},-\epsilon]^{\top}$ (i.e., the two vertices of the box $\mathcal{B}$ ), then $\mathcal{A}_{0}$ is UGAS for system (24).

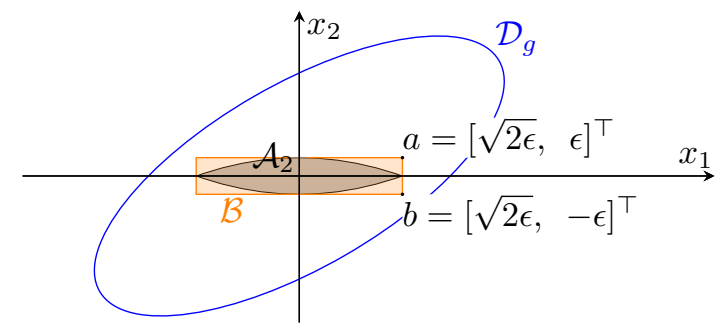

Fig. 2: Bounding box $\mathcal{B}$, attractor $\mathcal{A}_{2}$ and jump set $\mathcal{D}_{g}$.

\section{BOUNDED STABILIZATION OF THE ATTITUDE DYNAMiCS}

\section{A. Linearized model and input selection}

Consider the attitude dynamics of a rigid body parametrized by the unit quaternion $q=\left[\eta \varepsilon^{\top}\right]^{\top}$, where $\eta$ and $\varepsilon=\left[\begin{array}{lll}\varepsilon_{1} & \varepsilon_{2} & \varepsilon_{3}\end{array}\right]^{\top}$ are, respectively, the scalar and the vector component of the unit quaternion $q$ :

$$
\left\{\begin{aligned}
\dot{q} & =\frac{1}{2}\left[\begin{array}{c}
-\varepsilon^{\top} \\
\eta I_{3}+S(\varepsilon)
\end{array}\right] \omega \\
J \dot{\omega} & =S(J \omega) \omega+\tau
\end{aligned}\right.
$$

where

$$
S(x)=\left[\begin{array}{ccc}
0 & -x_{3} & x_{2} \\
x_{3} & 0 & -x_{1} \\
-x_{2} & x_{1} & 0
\end{array}\right]
$$

is the skew-symmetric cross-product operator acting on vector $x=\left[\begin{array}{lll}x_{1} & x_{2} & x_{3}\end{array}\right]^{\top} \in \mathbb{R}^{3}$ and satisfying $S(a) b=a \times b, J \in \mathbb{R}^{3 \times 3}$ is the inertia matrix of the rigid body, $\omega \in \mathbb{R}^{3}$ is the angular velocity of the rigid body expressed in the body frame, and $\tau=\left[\tau_{1} \tau_{2} \tau_{3}\right]^{\top} \in \mathbb{R}^{3}$ is the torque applied to the rigid body. Each component of $\tau$ cannot be larger than scalar $\bar{\tau}$ in norm, that is: $\tau_{i} \in[-\bar{\tau}, \bar{\tau}] \forall i \in\{1,2,3\}$.

Linearizing dynamics (28) around the unit quaternion $\left[\begin{array}{llll}1 & 0 & 0 & 0\end{array}\right]^{\top}$ with zero angular velocity allows us to write the attitude dynamics as follows:

$$
\dot{\zeta}=\left[\begin{array}{ll}
0 & 0 \\
I_{3} & 0
\end{array}\right] \zeta+\left[\begin{array}{c}
I_{3} \\
0
\end{array}\right] u
$$

where the components $u_{i}$ of $u, i=1,2,3$, must satisfy the normalized bound $\left|u_{i}\right| \leq 1$ and

$$
\zeta:=\frac{1}{\bar{\tau}}\left[\begin{array}{cc}
J & 0 \\
0 & 2 J
\end{array}\right]\left[\begin{array}{c}
\omega \\
\epsilon
\end{array}\right] \in \mathbb{R}^{6}
$$

From (29a), the linearized attitude dynamic around the unit quaternion with zero angular velocity can be represented by three decentralized double-integrator subsystems. We will refer to $\eta_{i}:=\left[\zeta_{i} \zeta_{3+i}\right]^{\top}$ as the $i$-th double-integrator subsystem. 
In controller (24), the local linear feedback has been embedded in the definition of matrix $A_{c}$ in (21), whereas in a real implementation the linear local feedback has to be made explicit.

To this end, each component of the input torque $\tau$ of the attitude dynamics (28) is interconnected to a realization of controller (24) as:

$$
\tau_{i}=\bar{\tau}\left((1-|q|) k \eta_{i}+\bar{\tau} q\right),
$$

where $\eta_{i}$ is the state of the $i$-th double integrator subsystem of the linearized attitude dynamics (29) defined above and $k$ is a stabilizing gain.

\section{B. Simulations}

This section shows simulations of plant (28) interconnected with three realizations of controller (24), leading to (30). The attitude dynamics data are chosen to fit with a commercial UAV:

$$
J=\left[\begin{array}{ccc}
3.1887 & 0 & 0 \\
0 & 3.2245 & 0 \\
0 & 0 & 1.3850
\end{array}\right] \mathrm{g} \mathrm{m}^{2}
$$

The torque saturation level $\bar{\tau}$ has been set to $0.15 \mathrm{Nm}$. The hysteresis parameter of the global controller $\epsilon$ has been set to $\epsilon=1 \cdot 10^{-4}$, while the hysteresis parameter of the united and local paradigm $\rho$ has been set to $\rho=0.5$. The local controller gain $k$ has been selected from an LQR synthesis on plant (29) and corresponds to $k=[101,100.00]$.

Two different initial conditions have been chosen. The first one, $\zeta_{01}$, is such that the system evolves near the linearization point of plant (28). The second one, $\zeta_{02}$, starts far away from the linearization point of the system. Such initial conditions are generated from a roll-pitch-yaw representation of the attitude. The choice of roll-pitch-yaw corresponding to $\zeta_{01}$ and $\zeta_{02}$ are reported in Table I. The initial angular velocity is supposed to be zero in both cases. The time responses are reported in Figures 3

\begin{tabular}{l|rr} 
& $\zeta_{01}$ & $\zeta_{02}$ \\
\hline roll & $-20^{\circ}$ & $20^{\circ}$ \\
pitch & $-20^{\circ}$ & $20^{\circ}$ \\
yaw & $20^{\circ}$ & $90^{\circ}$
\end{tabular}

TABLE I: Initial conditions Roll-Pitch-Yaw.

and 4. In both figures, the red line represents the evolution of the proposed control scheme with the selection of $\epsilon$ and $\rho$ reported above. In Figure 3, the thin black curve shows the evolution of the system starting from $\zeta_{01}$ but having set the hysteresis parameter $\epsilon=0$. It is possible to notice that such a selection turns into a chattering phenomena due to the unmodelled dynamics. Introducing the hysteresis in the global controller successfully removes the curve chattering.

In Figure 4, the thin black curve shows the evolution of the system starting from $\zeta_{02}$, but having set the hysteresis parameter $\rho=1$. It is possible to notice how such a selection turns into a slightly different chattering phenomenon in the first double-integrator subsystem with respect to the previous case (see Figure 4a). Around 0.1s, the solution seems to "stick" for a while, before continuing to evolve. A better understanding of this behavior is possible by looking at the phase portrait in Figure 5 comparing selections $\rho=1$ and $\rho=0.5$. The light-red filled area represents the set $\mathcal{C}_{g}:=\left\{\eta_{1} \mid \eta_{1}^{\top} P \eta_{1} \geq \rho \ell\right\}$ i.e., the set in which the global controller is active, while the light-blue filled area represents the set $\mathcal{C}_{l}:=\left\{\eta_{1} \mid \eta_{1}^{\top} P \eta_{1} \leq \ell\right\}$ i.e., the set in which the local controller is active. During the red portion of the orbit the global controller is active, while during the blue portion the local controller is active. From Figure 5 on the left we see that the solution sticks on the boundary of the sets $\mathcal{C}_{g}$ and $\mathcal{C}_{l}$, due to the absence of a hysteresis zone between the two sets because of the selection $\rho=1$, inducing high frequency switching between the local and global controller. Instead, in Figure 5 on the right, we see that 
an overlap of the sets $\mathcal{C}_{g}$ and $\mathcal{C}_{l}$, produced with a selection of $\rho=0.5$, (the light-purple filled doughnut) prevents this kind of behavior, resulting in a hysteresis zone that produces a faster convergence.

Nevertheless, from $\zeta_{02}$ the unmodelled dynamics is so relevant that the hysteresis in the global controller does not totally prevent the presence of unwanted switches.

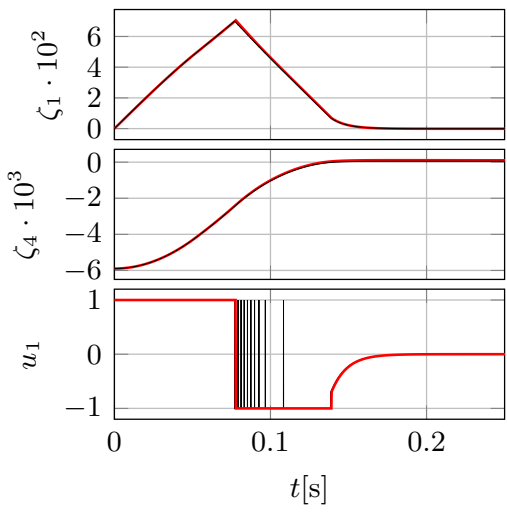

(a) First double-integrator subsystem.

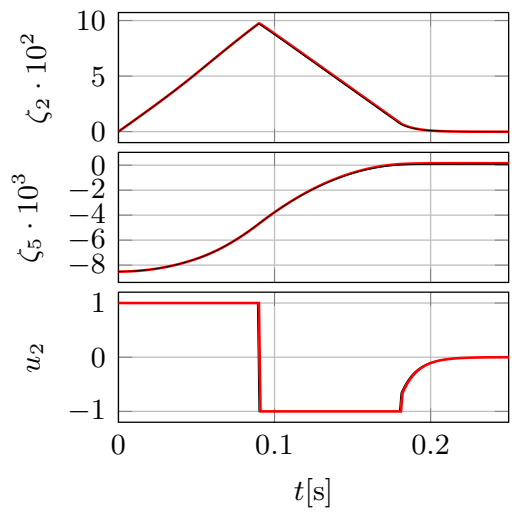

(b) Second double-integrator subsystem.

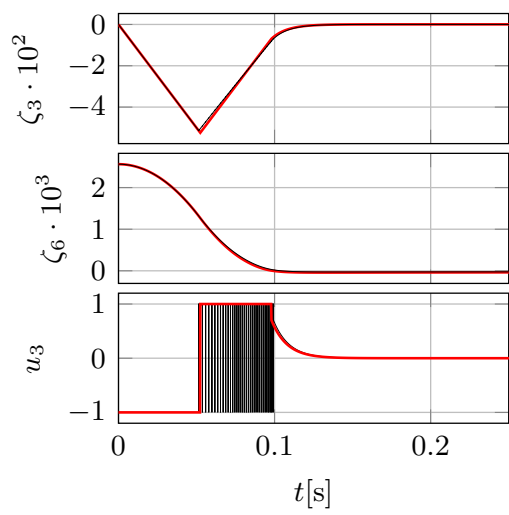

(c) Third double-integrator subsystem.

Fig. 3: Simulation results starting from $\zeta_{0}=\zeta_{01}$. Red: proposed controller. Black: removing hysteresis parameter $\epsilon$.

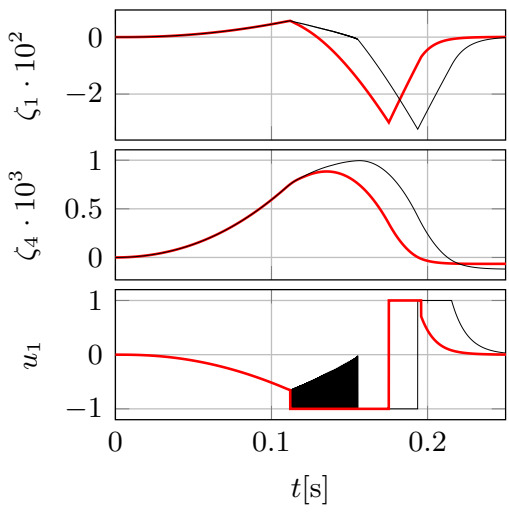

(a) First double-integrator subsystem.

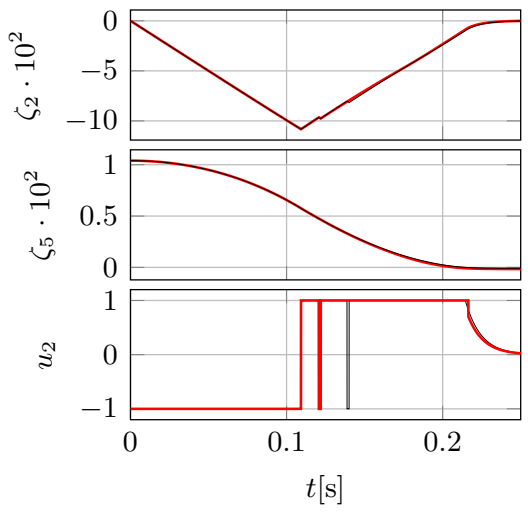

(b) Second double-integrator subsystem.

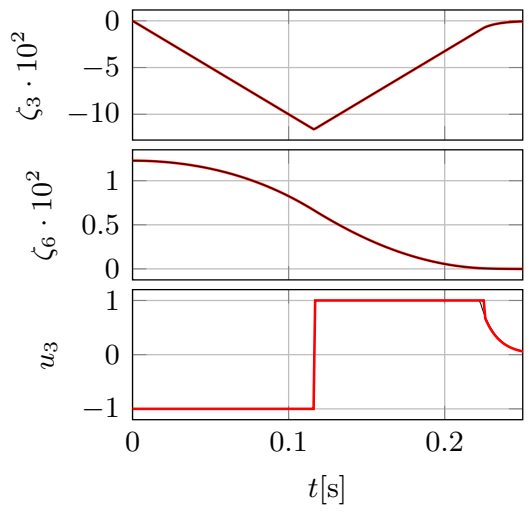

(c) Third double-integrator subsystem.

Fig. 4: Simulation results starting from $\zeta_{0}=\zeta_{02}$. Red: proposed controller. Black: removing hysteresis parameter $\rho$.

Both simulations show that the embedded hysteresis in the proposed control strategy is relevant in the presence of unmodeled dynamics (and/or in the presence of measurements noise), and prevents or reduces bad phenomena as chattering or sticking of the solution.

\section{CONCLUSIONS}

In this paper, we proposed a robust hybrid time-sub-optimal controller for the double integrator with saturated input. First, an analysis of the switching sets for the time-optimal control of a saturated double integrator has been carried out. Based on this description, an implicit hybrid framework for robust timesub-optimal control has been introduced. A hybrid blend with a local linear feedback has been then developed, which ensures global asymptotic stability of the origin.

Simulation results have shown the effectiveness of the proposed scheme for the attitude stabilization, in the presence of relevant unmodelled dynamics. In particular, the hysteresis mechanism in the global 

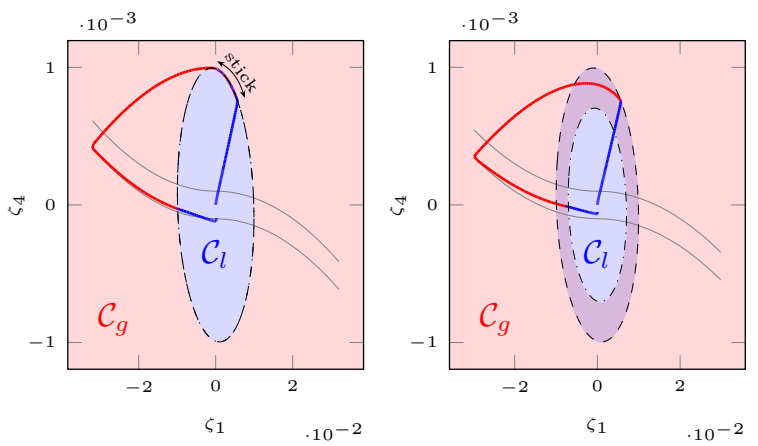

Fig. 5: Phase portrait of the first double-integrator subsystem starting from $\zeta_{0}=\zeta_{02}: \rho=1$ (left), $\rho=0.5$ (right).

controller and in the uniting global and local strategy has been shown to prevent chattering and sticking effects, producing clean and desirable evolutions.

Future research will include higher order systems, in particular the extension of the proposed scheme on the saturated triple integrator and further illustrating the technological advantages of the proposed stabilizers.

\section{REFERENCES}

[1] A. R. Teel, "Global stabilization and restricted tracking for multiple integrators with bounded controls," Systems \& control Lett., vol. 18, no. 3, pp. 165-171, 1992.

[2] L. Y. Pao and G. F. Franklin, "Proximate time-optimal control of third-order servomechanisms," IEEE Trans. Automatic Contr., vol. 38 , no. 4 , pp. 560-580, 1993.

[3] F. Forni, S. Galeani, and L. Zaccarian, "A family of global stabilizers for quasi-optimal control of planar linear saturated systems," IEEE Trans. Automatic Contr., vol. 55, no. 5, pp. 1175-1180, 2010.

[4] S. P. Bhat and D. S. Bernstein, "Finite-time stability of continuous autonomous systems," SIAM Journal on Control and Optimization, vol. 38, no. 3, pp. 751-766, 2000.

[5] M. Athans and P. L. Falb, Optimal control. An introduction to the theory and its applications. McGraw-Hill Book Co., New York-Toronto, Ont.-London, 1966.

[6] M. Brentari, A. Zambotti, L. Zaccarian, P. Bosetti, and F. Biral, "Position and speed control of a low-cost two-wheeled, self-balancing inverted pendulum vehicle," in Mechatronics (ICM), 2015 IEEE International Conference on, pp. 347-352, IEEE, 2015.

[7] H. S. Kim, S. Lim, C. C. Iuraşcu, F. C. Park, and Y. M. Cho, "A robust, discrete, near time-optimal controller for hard disk drives," Precision engineering, vol. 28, no. 4, pp. 459-468, 2004.

[8] U. Walther, T. T. Georgiou, and A. Tannenbaum, "On the computation of switching surfaces in optimal control: a Gröbner basis approach," IEEE Trans. Automatic Contr., vol. 46, no. 4, pp. 534-540, 2001.

[9] D. U. Patil and D. Chakraborty, "Computation of time optimal feedback control using Groebner basis," IEEE Trans. Automatic Contr., vol. 59, no. 8, pp. 2271-2276, 2014.

[10] W. Newman, "Robust near time-optimal control," IEEE Trans. Automatic Contr., vol. 35, no. 7, pp. 841-844, 1990.

[11] L. Y. Pao and G. F. Franklin, "The robustness of a proximate time-optional controller," IEEE Trans. Automatic Contr., vol. 39, no. 9, pp. 1963-1966, 1994.

[12] A. Hassibi, S. Boyd, and J. How, "A class of Lyapunov functionals for analyzing hybrid dynamical systems," in Proc. American Control Conference, pp. 2455-2460, 1999.

[13] V. G. Rao and D. S. Bernstein, "Setpoint tracking with actuator offset and sensor bias: probing the limits of integral control," IEEE Control Syst. Mag., vol. 21, no. 5, pp. 86-97, 2001.

[14] R. Goebel, R. G. Sanfelice, and A. R. Teel, Hybrid Dynamical Systems: modeling, stability, and robustness. Princeton University Press, 2012.

[15] S. van Loon, B. Hunnekens, W. Heemels, N. van de Wouw, and H. Nijmeijer, "Split-path nonlinear integral control for transient performance improvement," Automatica, vol. 66, pp. 262-270, 2016.

[16] D. Nešić, L. Zaccarian, and A. R. Teel, "Stability properties of reset systems," Automatica, vol. 44, no. 8, pp. $2019-2026,2008$.

[17] C. Prieur and A. R. Teel, "Uniting local and global output feedback controllers," IEEE Trans. Automatic Contr., vol. 56, no. 7, pp. 1636-1649, 2011.

[18] D. E. Kirk, Optimal control theory: an introduction. Courier Corporation, 2012.

[19] J. P. Hespanha, Linear systems theory. Princeton university press, 2009. 